\title{
GAP Production of TCM Herbs in China
}

Authors

Affiliations
Bengang Zhang ${ }^{1}$, Yong Peng ${ }^{1}$, Zhao Zhang ${ }^{1}$, Haitao Liu ${ }^{1}$, Yaodong Qi $^{1}$, Shuang Liu' ${ }^{2}$, Peigen Xiao ${ }^{1}$

${ }^{1}$ Institute of Medicinal Plant Development, Chinese Academy of Medical Sciences and Peking Union Medical College, Beijing, P. R. China

2 Certification Committee for Drugs, State Food and Drug Administration of the People's Republic of China, Beijing, P. R. China

\section{Key words \\ - GAP \\ - TCM herbs \\ - GAP base \\ - China \\ - cultivation}

\section{Abstract}

$\nabla$

In this paper, we briefly review international Good Agricultural Practice (GAP) regulations related to traditional Chinese medicine herbs (TCM herbs) and the background of the drafting process and the implementation of GAP for TCM herbs in China. We also have summarized progress and achievements since the implementation of GAP for TCM herbs in 2002. Up to 2010, a total of 99 GAP bases were formally adopted by GAP certification. They cover 22 provinces/municipalities, and 49 species of TCM herbs are currently cultivated in these GAP bases. Finally, we discuss the main problems in GAP implementation for Chinese crude drugs and put forward some suggestions to tackle them. received October 1,2010 revised October 13, 2010 accepted October 14, 2010

\section{Bibliography}

DOI http://dx.doi.org/

10.1055/s-0030-1250527

Published online November 12 , 2010

Planta Med 2010; 76 :

1948-1955 @ Georg Thieme Verlag KG Stuttgart · New York . ISSN 0032-0943

\section{Correspondence}

Prof. Peigen Xiao

Institute of Medicinal

Plant Development

Chinese Academy of Medical

Science and Peking

Union Medical College

No. 151, Malianwa North Road,

Haidian District

Beijing 100193

P.R. China

Phone: + 861063011294

Fax: + 861062899715

xiaopg@public.bta.net.cn

\section{Introduction}

$\nabla$

Traditional Chinese medicine (TCM), one of the treasures of the Chinese nation, has made great contributions to the development of both Chinese and world civilization for thousands of years. As traditional medicine and the research technology of TCM develop more rapidly than ever before, much attention is being paid to it worldwide because of its abundant resources, unique curative effects, low toxic and side effects, and more is being invested in its research and development while posing more demanding requirements for the quality of TCM products. However, many problems exist during the conventional TCM herbs production process in China, including unclear germplasm resources, substandard planting and processing techniques, excessive pesticide residue and poor quality of TCM herbs, thus leading to inconsistent quality of Chinese-made TCM products and severely affecting the stability of the curative effects of TCM as well as the lead position of Chinese TCM products in the world drug market. These challenges can be tackled in part through a Good Agricultural Practices (GAP) approach, which aims to standardize the cultivation, collection, and processing of TCM herbs, improving their quality and bringing TCM in line with the international practice, being therefore a key to the modernization and internationalization of TCM.

\section{GAP for TCM herbs}

GAP for TCM herbs, referred to as Chinese crude drugs (CCD) in Chinese official documents, is intended to control various factors affecting the production quality of medicinal plant materials, to standardize various crude drug production processes and even the whole process so as to ensure that TCM herbs are authentic, safe, effective, and consistent in quality [1]. Herein, TCM herbs cover CCD, herbal medicines, ethnodrugs, and introduced botanical drugs.

\section{Formulation of GAP for TCM herbs}

Along with the worldwide trend for returning to the nature, people are paying more attention to the medical and health care function of traditional medicines, especially TCM, and some countries have formulated relevant legislations concerning the quality control of herbal medicines.

Many countries have taken a series of standardized measures concerning quality control of production of raw materials for natural medicines. For example, the Japanese Ministry of Health, Labor, and Welfare revised Medicinal Plants Planting and Quality Evaluation in 1992; the US government issued a draft FDA Guidance for Industry: Botanical Drug Products in 1996; the European Herb Growers Association (Europam) proposed Good Quality Control of Medicinal Plants and Animals in August 1998; later, the European Union (EU) drafted Guidelines for Good Agricul- 
tural Practice (GAP) of medicinal and aromatic plants, commonly known as EU GAP, which were published officially in May 2002; the World Health Organization (WHO) began formulating guidelines on good agricultural and collection practices (GACP) for medicinal plants in August 2001 and published them in 2003. The international development trend of GAP indicates that its implementation is a common approach of various countries to ensure consistent quality and safety of medicinal plants or herbal medicines.

The State Food and Drug Administration (SFDA) of China put forward GAP for TCM herbs for the first time in November 1998. Based on domestic and foreign experiences in management of medicinal plants and animals, Good Agricultural Practice for Chinese Crude Drugs (Interim; "Chinese Crude Drug GAP") was officially published on April 17, 2002, and enacted as of June 1, 2002. In order to effectively implement GAP for TCM herbs, SFDA formulated and enacted the "Management Measures of Chinese Crude Drug GAP Certification" (Provisional) and "Chinese Crude Drug GAP Certification Evaluation Criteria" (Provisional) on September 19, 2003, which symbolized the commencement of the standardization of TCM herb production in China.

\section{Significance of GAP for TCM herbs}

TCM herbs are the basic material of the decoction pieces and for the production of Chinese traditional patent medicines. Safety and consistent quality of TCM herbs is the physical prerequisite for the clinic application of TCM. GAP for TCM herbs is intended to control their quality during the whole process of production and ensure their safety, consistent quality, and controllability. The safety and consistent quality of TCM herbs also provides solid assurance for GLP, GCP, GMP, and GSP of TCM. GAP is therefore a requisite to standardize and modernize TCM. It also is the key to enhance international competitiveness of TCM and to realize its internationalization.

The GAP approach is one of the important measures to the industrialization of TCM herb agriculture. Standardization, commercial scale, and industrialized production of TCM herbs are a prerequisite for their use as pharmaceuticals and in health care. It also is a way to improve rural livelihoods and income [1]. Furthermore, the collections of wild resources are standardized in the implementation of GAP, which plays a role in protecting wild herb resources and achieving the sustainable use of TCM [2].

\section{Overview of GAP for TCM herbs}

GAP implementation is to systematically regulate the whole process of production, controlling the quality of TCM herbs in order to achieve their standardization. It involves the following eight parts [1].

Ecological environment of the production site: At the production base, producers should rationally select their production sites based on local conditions of soil fertility, soil contaminants, cultivation history, location and setting of the site, with special attention given to the recommended production area of geo-authentic crude drugs (Dao Di). A total of 99 bases of GAP for TCM herbs ( 49 species) were certified before 2010 by SFDA (see 0 Table 1 ). The choice of the bases to plant TCM herbs by the GAP approach followed aspects related to GAP for the TCM herbs under consideration, that is, natural distribution area of the original species, cultivation and domestication history, the level of socioeconomic development, human culture, and social environment. For safety's sake, requirements have been imposed on the quality of soil, farm irrigation water, and animal drinking water of the pro- duction sites. The environmental conditions of the production sites should meet the requirements of the related national standards: "Atmospheric Conditions Standard (GB 3095-1996)", Grade 2 for air quality; "Soil Quality Standard (GB 15628-1995)", Grade 2 for soil; "Farm Irrigation Water Standard (GB 5084-92)", Grade 2 for irrigation; "Drinking Water Standard (GB 5749-85)" for animal drinking water.

Germplasm and propagation materials: At first, propagation materials should be identified accurately as to their relevant taxonomic rank. Homonyms and synonyms of TCM herbs are very common in traditional Chinese medicine. For example, the original plant of Dang Gui is Angelica sinensis in the Pharmacopoeia of the PRC. However, Dang Gui involves 8 species of 2 families in folk and traditional usage, while there are 11 vernacular names of A. sinensis in traditional literature and local nomenclature systems. Therefore, the adopted and Latin names of TCM herbs should be clearly recorded to avoid nomenclatural confusion. Secondly, the germplasm of propagation materials should be paid more attention to in practice. Many cultivars of medicinal plants with good agronomic traits have been selected and bred during the long cultivation history of China, such as Rehmannia glutinosa cv. "Jin Zhuang Yuan" and R. glutinosa cv. "Xiao Hei Ying". They have significant differences in stress resistance and yield. Finally, when certain species have different secondary metabolites in different populations, the propagation materials should be well chosen. The reason lies in the fact that different ecological factors could cause diverse secondary metabolic pathways. For example, Securinega suffruticosa in the Beijing population contains $d$-securinine, while $l$-securinine appears in the northeastern China population, and the two kinds of securinnine coexist in the Chengde population.

Cultivation and feeding management: TCM herb cultivation management demands formulating standard operating procedures (SOPs) for the production technology. The contents of SOPs include a fertilizing program, irrigation technology, pest control measure, determination methods, and limitation standards of toxic and harmful substances in TCM herbs, as well as application of specific fertilizers for TCM herb cultivation and pesticide residue standards. Medicinal animal feeding and management also require developing SOPs, including the condition of facilities, health management, feed, feed additives, drinking water, disease prevention and control. The book of "Guidelines for Good Agricultural Practice of Chinese Crude Drugs", which is equivalent to an official publication, provides the SOPs model of 4 species, including Panax notoginseng, Lycium barbarum, Glycyrrhiza uralensis, and Houttuynia cordata [3].

Harvesting and primary processing: Requirements are formulated on the harvesting period, harvesting equipment and processing sites, and post-harvest processing techniques. SOPs for harvesting and processing are required to be formulated. There are 12807 species being used as medicinal plants, animals, and fungi in China. Only 400 species of them are cultivated and domesticated, most of them are from wild resources. Therefore, wild medicinal resource utilization should be well planned according to the principle of sustainable use. Such methods as wild fostering, interval harvesting, planting young seedlings to replace harvested herbs, etc., should be adopted to achieve the benefits both of medicinal resource conservation and economic interest. Based on scientific research and traditional experiences, any parts of plant and animal organ or secretion could be used as TCM herbs depending on where the effective medicinal ingredients are accumulated. Collected parts should be not arbitrarily altered in 
Table 1 GAP production bases and species of TCM herbs adopted by national certification before 2010 in China.

\begin{tabular}{|c|c|c|}
\hline Species & Locality & Province \\
\hline Fritillaria ussuriensis Maxim. & 1 Yichun City & \\
\hline Fritillaria ussuriensis Maxim. & 2 Tieli City & Heilongjiang \\
\hline Isatis indigotica Fort. & 3 Daqing City & \\
\hline Panax ginseng C. A. Mey. & 4 Jingyu County & \\
\hline Panax quinquefolius L. & 5 Jingyu County & \\
\hline Panax ginseng C. A. Mey. & 6 Fusong County & Jilin \\
\hline Panax ginseng C. A. Mey. & 7 Linjiang City & \\
\hline Panax ginseng C. A. Mey. & 8 Changbai County & \\
\hline Panax ginseng C. A. Mey. & 9 Ma County & \\
\hline Gentiana scabra Bge. & 10 Qingyuan County & Liaoning \\
\hline Schisandra chinensis (Turcz.) Baill. & 11 Xinbin County & \\
\hline Astragalus membranaceus (Fisch.) Bge. var. Mongholicus (Bge.) Hsiao & 12 Qahar Youhouqi & Inner Mongolia \\
\hline Isatis indigotica Fort. & 13 Yutian County & \\
\hline Schizonepeta tenuifolia Briq. & 14 Yutian County & Hebei \\
\hline Corydalis bungeana Turcz. & 15 Yutian County & \\
\hline Astragalus membranaceus (Fisch.) Bge. var. Mongholicus (Bge.) Hsiao & 16 Huiyuan County & Shanxi \\
\hline Sophora flavescens Ait. & 17 Qin County & \\
\hline Papaver somniferum L. & 18 Zhangye City & \\
\hline Papaver somniferum L. & 19 Zhangye City & \\
\hline Papaver orientale L. & 20 Jingchang City & \\
\hline Papaver somniferum $\mathrm{L}$. & 21 Jingchang City & \\
\hline Papaver somniferum Lindl. & 22 Wuwei City & Gansu \\
\hline Papaver somniferum L. & 23 Wuwei City & \\
\hline Papaver somniferum L. & 24 Baiyin City & \\
\hline Papaver somniferum $\mathrm{L}$. & 25 Baiyin City & \\
\hline Angelica sinensis (Oliv.) Diels & 26 Min County & \\
\hline Angelica sinensis (Oliv.) Diels & 27 Dangchang County & \\
\hline Platycodon grandiflorus (Jacq.) A. DC. & 28 Yiyuan County & Shangdong \\
\hline Lonicera japonica Thunb. & 29 Pingyi County & \\
\hline Rehmannia glutinosa Libosch. & 30 Wen County & \\
\hline Dioscorea opposita Thunb. & 31 Wen County & \\
\hline Rehmannia glutinosa Libosch. & 32 Wushe County & \\
\hline Dioscorea opposita Thunb. & 33 Wushe County & \\
\hline Lonicera japonica Thunb. & 34 Fengqiu County & Henan \\
\hline Cornus officinalis Sieb. et Zucc. & 35 Xixia County & \\
\hline Cornus officinalis Sieb. et Zucc. & 36 Xixia County & \\
\hline Cornus officinalis Sieb. et Zucc. & 37 Neixiang County & \\
\hline Isatis indigotica Fort. & 38 Taihe County & Anhui \\
\hline Ginkgo biloba L. & 39 Pizhou City & Jiangsu \\
\hline Codonopsis pilosula (Franch.) Nannf. & 40 Lingchuan County & \\
\hline Salvia miltiorrhiza Bge. & 41 Luonan County & \\
\hline Salvia miltiorrhiza Bge. & 42 Danfeng County & \\
\hline Salvia miltiorrhiza Bge. & 43 Zuoshui County & \\
\hline Salvia miltiorrhiza Bge. & 44 Shanyang County & \\
\hline Cornus officinalis Sieb. et Zucc. & 45 Foping County & Shaanxi \\
\hline Salvia miltiorrhiza Bge. & 46 Shangnan Count & \\
\hline Salvia miltiorrhiza Bge. & 47 Zhen'an County & \\
\hline Gastrodia elata BI. & 48 Lueyang County & \\
\hline Gynostemma pentaphyllum (Thunb.) Makino & 49 Pingli County & \\
\hline Crocus sativus L. & 50 Chongming County & Shanghai \\
\hline Crocus sativus L. & 51 Baoshan District & \\
\hline Aconitum carmichaelii Debx. & 52 Jiangyou City & \\
\hline Ligusticum chuanxiong Hort. & 53 Wenchuan County & \\
\hline Ophiopogon japonicus (Thunb.) Ker-Gawl. & 54 Santai County & \\
\hline Angelica dahurica (Fisch. ex Hoffm.) Benth. et Hook. f. & 55 Shehong County & \\
\hline Angelica dahurica (Fisch. ex Hoffm.) Benth. et Hook. f. & 56 Pengxi County & Sichuan \\
\hline Ligusticum chuanxiong Hort. & 57 Pengzhou City & \\
\hline Angelica dahurica (Fisch. ex Hoffm.) Benth. et Hook. f. & 58 Suining City & \\
\hline Houttuynia cordata Thunb. & 59 Ya'an & \\
\hline Houttuynia cordata Thunb. & 60 Ya'an & \\
\hline Periplaneta americana L. & 61 Xichang City & \\
\hline Tussilago farfara $\mathrm{L}$. & 62 Wuxi County & \\
\hline Coptis chinensis Franch. & 63 Shizhu County & Chongqing \\
\hline Artemisia annua L. & 64 Youyang County & \\
\hline
\end{tabular}


Table 1 GAP production bases and species of TCM herbs adopted by national certification before 2010 in China. (continued)

\begin{tabular}{|c|c|c|}
\hline Species & Locality & Province \\
\hline Scrophularia ningpoensis Hemsl. & 65 Badong County & Hubei \\
\hline Scrophularia ningpoensis Hemsl. & 66 Jianshi County & \\
\hline Cornus officinalis Sieb. et Zucc. & 67 Lin'an County & \\
\hline Cornus officinalis Sieb. et Zucc. & 68 Chun'an County & \\
\hline Dendrobium officinale Kimura et Migo & 69 Tiantai County & Zhejiang \\
\hline Coix lacryma-jobi L. var. ma-yuen (Roman.) Stapf & 70 Taishun County & \\
\hline Gardenia jasminoides Ellis & 71 Zhangshu County & \\
\hline Corydalis yanhusuo W. T. Wang & 72 Fuzhou City & Jiangxi \\
\hline Gardenia jasminoides Ellis & 73 Xingan City & \\
\hline Pseudostellaria heterophylla (Miq.) Pax ex Pax et Hoffm. & 74 Zherong County & \\
\hline Alisma orientalis (Sam.) Juzep. & 75 Jian'ou City & Fujian \\
\hline Polygonum multiflorum Thunb. & 76 Cengong County & \\
\hline Polygonum multiflorum Thunb. & 77 Shibing County & \\
\hline Pseudostellaria heterophylla (Miq.) Pax ex Pax et Hoffm. & 78 Shibing County & \\
\hline Polygonum capitatum Buch. -Ham. ex D. Don & 79 Shibing County & Guizhou \\
\hline Pseudostellaria heterophylla (Miq.) Pax ex Pax et Hoffm. & 80 Huangping County & \\
\hline Epimedium wushanense T.S.Ying & 81 Xiuwen County & \\
\hline Polygonum multiflorum Thunb. & 82 Jinping County & \\
\hline Polygonum multiflorum Thunb. & 83 Kaili County & \\
\hline Pseudostellaria heterophylla (Miq.) Pax ex Pax et Hoffm. & 84 Kaili County & \\
\hline Polygonum capitatum Buch. -Ham. ex D. Don & 85 Guiyang City & Guizhou \\
\hline Epimedium wushanense T.S.Ying & 86 Longli Coutny & \\
\hline Pseudostellaria heterophylla (Miq.) Pax ex Pax et Hoffm. & 87 Leishan County & \\
\hline Epimedium wushanense T.S.Ying & 88 Leishan County & \\
\hline Polygonum multiflorum Thunb. & 89 Congjiang County & \\
\hline Erigeron breviscapus (Vant.) Hand.-Mazz. & 90 Luxi County & \\
\hline Aucklandia lappa Decne. & 91 Yulong County & \\
\hline Panax notoginseng (Burk.) F. H. Chen & 92 Liangshan County & \\
\hline Panax notoginseng (Burk.) F. H. Chen & 93 Wenshan County & Yunan \\
\hline Panax notoginseng (Burk.) F. H. Chen & 94 Wenshan County & \\
\hline Panax notoginseng (Burk.) F. H. Chen & 95 Maguan County & \\
\hline Panax notoginseng (Burk.) F. H. Chen & 96 Maguan County & \\
\hline Andrographis paniculata (Burm. f.) Nees & 97 Yingde City & \\
\hline Pogostemon cablin (Blanco) Benth. & 98 Guangzhou City & Guangdong \\
\hline Pogostemon cablin (Blanco) Benth. & 99 Suixi County & \\
\hline
\end{tabular}

the collection process. Appropriate collection time (season and years) and methods should be determined in accordance with the quality and yield of the plants. For example, the best collection time of Panax ginseng is at the sixth year of growth, which is proved by research comparing the accumulating value of the effective ingredient and yield of the medicinal part annually. Machines and tools for collection should be kept clean and free of contamination, and stored in a dry place inaccessible for insects, rodents, poultry, and livestock. In the course of collection and primary processing, nonmedicinal portions and foreign matter should be removed, especially weeds and toxic substances. Damaged and perished parts should be excluded. After being collected, the medicinal parts should be selected, washed, cut, or trimmed. Those that need drying should be dried timely by using appropriate methods and techniques, with controlled temperature and humidity. The use of antioxidant agents and preservatives should be avoided. If used, they must conform to the national regulatory requirements on food additives (GB 2760-1996). Processing sites should be clean and well ventilated and fitted with awnings, canopies, and devices to prevent the entry of insects, rodents, poultry, and livestock. Geo-authentic crude drugs should be processed according to traditional methods. Any change in methods should be based on sufficient experimental data, and should not affect the quality of the TCM herbs.
Packaging, transportation, and storage: Specific requirements have been defined for packaging materials and methods, condition of the transporting containers and the warehouse for crude drug storage. Prior to packaging, crude drugs should be checked and substandard ones as well as foreign matter should be eliminated. The packaging materials to be used should be clean, dry, uncontaminated and undamaged, and conform to the quality requirements for crude drugs. On each package of the crude drugs, the product name, specification, production site, batch number, packaging date, and the name of producer should be indicated and a sign for qualified products should be marked. Fragile crude drugs should be packaged in hard boxes. Poisonous/toxic, narcotic, and precious crude drugs should be specially packaged, and appropriately marked. The crude drugs should not be put together with poisonous/toxic, hazardous, and volatile materials during their transportation. Containers should be well-aerated to keep the crude drugs dry, and moisture proof measures should be in place. The warehouse for crude drugs should be airy and dry, and it should protect the crude drugs from direct sunlight. If necessary, air conditioners and dehumidifiers should be installed. Measures to prevent entry into the warehouse from insects, rodents, poultry, and livestock should be taken. Floors should be neat and tidy, free from cracks and easy to clean. The crude drugs should be placed on shelves and kept at an adequate distance from the wall, with avoidance of moth-eating, mould 
growth, rotting, and oil spillage. Regular checks should be conducted.

Quality management: Quality inspection is conducted according to the standards for TCM herbs approved by the government, including Pharmacopoeia of the PRC and Drug Standard of Ministry of Public Health of the PRC. Those TCM herbs without quality inspection of national standards should comply with related standards of local governments. The testing items should at least cover macroscopic characters and identification of crude drugs, impurities, moisture content, total ash content and acid insoluble ash content, extracts and efficacious contents. Pesticide residue, heavy metal, and microbiological limits should comply with the Green Standards of Medicinal Plants and Preparations for Foreign Trade and Economy of Ministry of Commerce of the PRC.

Personnel and facilities: The responsible persons for technology and quality management of a producer are the key personnel for implementing GAP for TCM herbs. They should meet the requirements for educational background, qualification, professional knowledge, and capability to resolve practical issues and problems in production and quality management processes. Requirements should also be raised for the facilities of producers, instruments, and equipment for production and inspection.

Documentation: Management and SOPs, quality standards, testing rules, and quality control procedures should all be documented in detail and distributed to the relevant user and operators for training and implementation. These documents are targeted to ensure GAP implementation in a dynamic and orderly manner, as well as traceability of quality management of TCM herbs.

\section{Latest Developments of GAP Production of TCM Herbs in China}

$\nabla$

Generous support was given by the Chinese central government and great achievement was acquired

Eight years have elapsed since the enactment of the Good Agricultural Practice for Chinese Crude Drugs (Interim). The Chinese central government has been given generous support to implement GAP for TCM herbs, resulting in rapid development of GAP for TCM herbs and the Chinese TCM industry. During this period, the government financially supported technical research of standardized planting (breeding) of 182 species of TCM herbs with the Key Technologies R \& D Programme of the 9th and 10th fiveyear plan [4]. In addition, standardized planting of TCM herbs and a related research program have been sponsored by the National Development and Reform Commission, State Administration of Traditional Chinese Medicine, Ministry of Science and Technology. Owing to the generous support, there is remarkable progress on technical research of standardized planting of TCM herbs, and several influential books were published, such as Guidelines for Good Agricultural Practice for Chinese Crude Drugs. GAP production base of TCM herbs certification, as the key step of GAP implementation, has effectively mobilized enterprises to implement GAP since the "Management Measures of Chinese Crude Drug GAP Certification" were approved by the State Council. So far, 22 provinces/municipalities have established standardized TCM herb planting bases. Up to 2010, a total of 99 GAP bases were formally adopted by GAP Certification. They cover 22 provinces/municipalities, and 49 species of TCM herbs are currently cultivated in these GAP bases (see $\bullet$ Fig. 1 and Table 1). The area of plantations accredited is growing rapidly. For ex- ample, in Jilin Province, there are 20000 ha central planting area and 43000 ha demonstration planting area, more than five times that of 1995 [5]. GAP base certification effectively addresses the problem of substandard medicinal material production in China and helps to bring medicinal material production onto the track of standardization.

\section{GAP implementation is widely recognized by TCM} enterprises and farmers

Implementation of GAP for TCM herbs had a tremendous impact on people's traditional ideas, so that the understanding of "traditional Chinese medicine production is extensive small-scale farmer's production" has been completely changed. So people started paying more attention to the quality of TCM herbs, and the importance of their standardized production has become more salient. Implementation of GAP has changed the TCM herb production practice characterized formerly by disorderly development, lack of government regulation, and extensive farming that remained since the 1980s. It resolved many longtime problems with TCM herb production activities, such as germplasm resource background confusion, substandard production processes, and abuse of pesticides and chemical fertilizers.

\section{Industrialization of TCM herbs was catalyzed \\ by GAP implementation}

Standardization became the mainstream aspect of TCM herb production bases. According to preliminary statistics, Sichuan, Shaanxi, Gansu, Yunnan, Jilin, Henan, Anhui, and Guangdong provinces have completed more than 50 standardized TCM herb production bases. Meanwhile, medium- and large-sized pharmaceutical industry establishments began building their own medicinal material plantations, which rapidly drove production of TCM herbs towards industrialization and effectively drew dispersed farmers together through market mechanisms. Thus, a modern agricultural production model consisting of company + plantation + farmers was introduced into the TCM herb production practice, thereby effectively facilitating intensive and largescale production of TCM herbs in the country and resulting in rapid and sound development of TCM herb production [4].

\section{Wild tending techniques were applied in GAP for TCM herbs $\nabla$}

At present, about $80 \%$ of medicinal materials are still derived from the wild in China [6]. Along with the implementation and promotion of GAP for TCM herbs, a number of medicinal materials which formerly had to rely on wild resources, such as Gastrodia elata, Pinellia ternata, Bupleurum chinense, Gentiana scabra, Ledebouriella divaricata, Schisandra chinensis, Citrus grandis, Tripterygium wilfordii, Pueraria lobata, Glycyrrhiza uralensis, Ephedra sinica, Cistanche deserticola, Saussureae involucratae, etc. [4,7] have been introduced and cultivated on a large scale. The technology for wild tending, such as Fritillaria cirrhosa and Cordyceps sinensis, has been getting mature; GAP base construction has been rapidly developed. The plantations of Gastrodia elata, Gentiana scabra, and Schisandra chinensis have been adopted by national certification of GAP production bases of TCM herbs. 


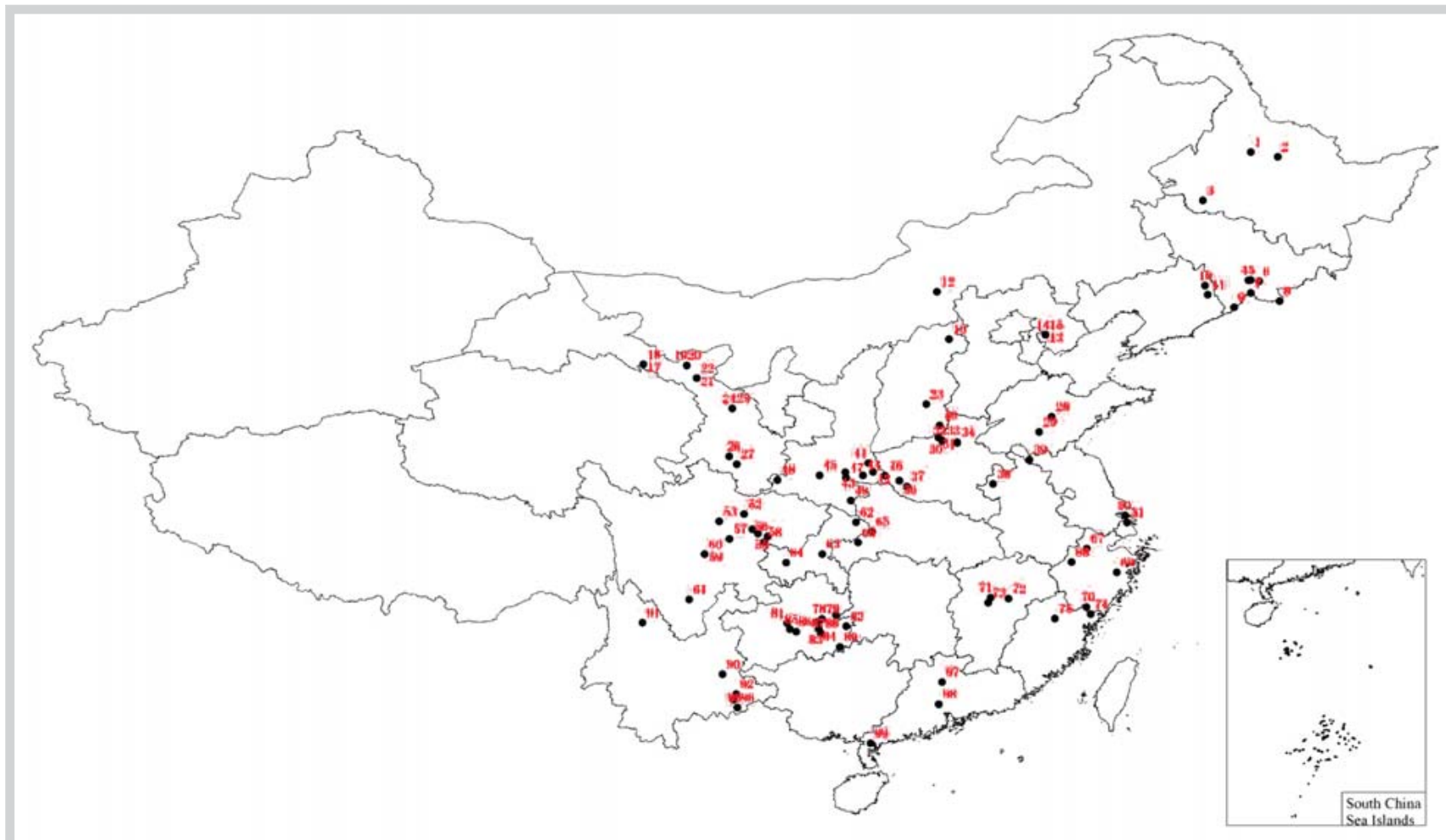

Fig. 1 GAP production bases of TCM herbs adopted by national certification before 2010 in China.

\section{Gradual internationalization of TCM drew} attention from the international medical fields Through seven years' implementation and spread of GAP, many countries began understanding and accepting TCM, leading to a steady increase in the export of TCM products from China with a sum of 1 billion dollars up to now [8]. Currently, over 120 countries around the world are using traditional medicines, and some European countries specify some medicinal plants that are permitted to be sold in drugstores as medicines or OTC medicines in their relevant legislations. U.S. FDA brought forward a proposal to declare plant products as drugs, laid down legislation on herbal medicines and included herbal medicines in medical insurance as therapeutic drugs instead of nutrients or substitute drugs [2]. In April 2004, the European Parliament and European Council adopted a revised law concerning the traditional medicinal products. Over 70 countries around the world have formulated herbal medicine-related legislations [9].

\section{Problems with Implementation of GAP for TCM Herbs in China \\ $\nabla$}

Expanding scale of plantation and reckless introduction of species from the outside leads to lower quality of medicinal materials

Reckless expansion of the scale of plantation is one of the serious problems in the cultivation of TCM herbs at present, actually resulting from lack of marketing concepts and unified planning and coordination. The traditional small-scale farming practice and blind trend following among farmers are the main reasons why the TCM herb market fluctuates. Ignoring the rule of market economy and recklessly expanding the scale of plantation may result in vicious competition, disrupt the market and lower the quality of medicinal materials resulting in the frustration of farmers.

Introduction of nonnative species is another common problem of the present production of TCM herbs. An essential concept involved in production of TCM herbs are geo-authentic crude drugs (Dao $\mathrm{Di}$ ). But this concept has been ignored when nonnative species became prevalent across the country for expansion of production scale and greater economic benefits. For example, because of high medicinal value and considerable economic benefit, Panax ginseng was introduced from its geo-authentic production areas, northeast China, to Yunnan province of southwest China in the 1970s. Although the ginseng product of Yunnan province showed good agronomic traits, the low content for ginsenosides resulted in its poor medicinal value. These reckless practices destroyed the requirements of geo-authenticity and then lowered the quality of TCM herbs by violating the law of nature.

\section{Operational model of company + plantation + farmers interaction needs to be improved}

Currently, most of TCM herb plantations in the country run the operational model of company + plantation + farmers, in which the company usually cooperates with research institutes to provide technical support and guide the whole process of production management of TCM herbs. However, the economically underdeveloped background of rural areas, especially some remote rural areas, where the active labor force rushes to cities for making a living and only the illiterate senior citizens and the weak are left behind, make it very difficult to realize standardized production management and to ensure that farmers can strictly follow GAP requirements even with technical training $[10,11]$. How to organize numerous farmers to realize standardized and large-scale 
production of TCM herbs strictly according to GAP requirements remains to be explored in practice.

\section{Basic research of key production technology is weak}

Research of biological characteristics and cultivation techniques of TCM herbs is still at the infant stage. Deep studies of growth and developmental characteristics of various medicinal crops are absent, and growth regulation, quality, and yield formation as well as formulation of growth control measures lack a theoretical basis. In particular, farmers in traditional producing areas mainly rely upon conventional planting experiences, and the production control measure lacks a scientific basis and verification. In addition, only attaching importance to the production and neglecting document management, archives, original records, and traceability, highlighted requirements in the GAP, are also common problems.

\section{Supporting policy and quality standard system are imperfect}

So far, the government has not yet enacted any provisions to enforce implementing GAP for TCM herbs, nor included GAP in pharmaceutical management law. Moreover, there is no official regulation that the raw materials according to GMP, GLP, GCP, or GSP should come from GAP production or designated supply bases. As GAP production of TCM herbs will inevitably result in increased cost and investment, the GAP implementation process will be unsustainable if there is no supporting government policy to ensure the higher costs.

According to GAP for TCM herb requirements, heavy metal and pesticide residues should be strictly controlled to an acceptable level. But there is neither a statutory upper limit of heavy metal or pesticide residue contained in TCM herbs nor a specific pesticide monitoring agency. Now the Pharmacopoeia of the PRC (2010) only contains heavy metal and pesticide residue limits for Radix Glycyrrhizae and Radix Astragali.

\section{Measures and Suggestions}

$\nabla$

\section{GAP base construction and species to be cultivated}

should respect the local conditions and regular pattern

of economy

Production of TCM herbs is highly specific to geographic location, and a particular ecological environment is one of the prerequisites for the production of high-quality medicinal plant materials. When building GAP bases, it is necessary to respect local conditions, selecting appropriate places, to choose suitable soil and a geographic environment based on the ecological and biological characteristics of each kind of TCM herb [12]. Equal emphasis should be placed on the geography of geo-authentic crude drugs and the place of origin [13].

Geo-authentic crude drugs are those commonly recognized by the general public for their long history, high quality, and curative effect; they are produced under certain natural conditions using particular production technology and processing techniques. Geo-authentic crude drugs are usually named after their places of origin, for example, Sichuan Coptis chinensis (Chuan Huang Lian), Zhejiang Fritillaria thunbergii (Zhe Bei Mu), Liaoning Schisandra chinensis (Liao Wu Wei), and Henan Dioscorea opposita (Huai Shan Yao) [1]. Suitable ecological environment, excellent germplasm resources, and time-tested production and processing techniques are the basic elements in formation and develop- ment of geo-authentic crude drugs [14]. The generation of geoauthentic crude drugs is a product of interaction between genotype and environment from the biological perspective [15]. Although the regulations of GAP for TCM herbs do not stipulate that TCM herbs have to be cultivated in the place of origin, it specifies that a "suitable location of cultivation shall be determined according to the requirements of growth and development of medicinal plants." Therefore location of base and germplasm of TCM herbs should be selected strictly according to place of origin and suitability of the species or cultivars so as to ensure the high quality of TCM herbs.

Blindly expanding the scale of plantation of TCM herbs and ignoring the preliminary market survey led to vicious competition and low quality and were a blow to the enthusiasm of farmers. For example, the cultivation area of Schisandra chinensis increased 40 times from 2000 to 2007, but its market price reduced $85 \%$ during this period [16]. Therefore, in the development of a planting plan, it is necessary to determine the size of areas planted to be targeted and do premarket research to avoid economic losses.

\section{Optimize the operational model of GAP bases}

Development of GAP bases of TCM herbs should be guided by scientific technology facilitated by standardization and aimed at industrialization and should follow the demands of the market and center on enterprises. The industry chain of GAP for TCM herbs should be established, and suitable operational models should be determined taking into account local conditions in order to emphasize the role of GAP production of TCM herbs in promoting TCM industry and social and economic progress.

The operational model of company + plantation + farmers is based on high competency of practitioners and effective technical training, which is a prevailing model of GAP for TCM herbs at present in China. The formation of this model is mainly due to the economic structure in rural areas, a labor allocation system, and the natural environment of mountainous areas. This model is not the best one from the perspective of standardization as required by GAP. Instead of the above-mentioned model, rural cooperatives, intensive farming and plantation, and specialized corporate planters will become the main direction of standardization of TCM herbs production along with economic development. Given the current conditions, the operational model of company + plantation + farmers should be improved by enhancing the competency and qualification of practitioners, raising the awareness of farmers about standardization, and conducting technical training.

\section{Increase investment in scientific research} and formulate practical SOP

One of the authors, Prof. Bengang Zhang, has participated as an inspector in nearly 20 GAP certifications of plant production bases and found a lot of SOPs were not precise enough and had a poor operability, which was directly related to the intensity of research and development investments, including investment in scientific and technological personnel and research funds input. Therefore, the establishment of a GAP base for TCM herbs should stimulate the basic scientific research into TCM to solve some technical problems in the production of GAP for TCM herbs. It should ensure high-quality conditions and fully enhance the production of plant medicines. With the direction of "Good Agricultural Practice (Interim)" (referred to as GAP for TCM herbs), the production base should be in accordance with their actual situation of production of medicinal plant species and meet the re- 
quirements for developing methods and measures leading to SOPs. SOPs should be formulated through scientific research and experimentation and have scientific, complete, practical, and rigorous characteristics. Their content should include the entire production, processing, quality control, packaging, transport, and storage of the entire process.

\section{Enterprises should be responsible for developing and improving the relevant quality standards}

TCM herb production enterprises should develop enterprise standards based on national and local government standards to control the quality of their own TCM herb production. They should at least include determination of active ingredients and marker components, limit standards of hazards, heavy metal and pesticide residues, and microbiological purity.

\section{Conclusion}

\section{$\nabla$}

Implementation of GAP for TCM herbs has made a remarkable contribution to the modernization of TCM. It had profound influence on the production of TCM herbs and development of TCM, and conformed to the international trend of production of TCM herbs. It should accelerate the steps of standardization, intensive management, and internationalization of TCM herbs. It should promote the adjustment of agriculture structure and development of TCM agriculture. It should improve the quality of medicinal herbs and market competitiveness of TCM herbs.

However, GAP for TCM herbs is a new development in China, and the production of TCM herbs is mainly undertaken by thousands of farmers scattered throughout the country. Implementation of GAP for TCM herbs is a systemic project, including multiple disciplines, social conditions, and the farmer's initiative. Therefore, full implementation of GAP for TCM herbs still has a very long way to go, and many issues need to be improved in the implementation process.

In the process of implementation of GAP, the following points should be paid more attention to:

Up to now, there are over 400 species of TCM herbs being cultivated in China [17]. Owing to diverse growth characteristics and cultivation technologies in such large number of TCM herbs, relevant basic researches need to be improved.

Explore the operational management mode of production of TCM herbs, encourage the producer to approach large-scale production so as to achieve the purpose of uniform quality of products. Recommend establishing production bases in the geo-authentic production regions because the TCM herbs produced there were recognized widely as the result of good quality proved in the long-term application history. The skillful cultivation experience of local producers makes it easy to establish SOPs based on the traditional production experience and processing methods, so as to ensure the geo-authenticity of TCM herbs.

In the process of GAP implementation, some technicians have a weak knowledge related to GAP leading to the inadequate performance of SOPs. So it is necessary to strengthen the theoretical and technical training of producers to improve their operating and managing skill.

In summary, implementation of GAP for TCM herbs is an important measure to ensure the quality of TCM herbs. It can not only meet the demands of consumers and retailers, but also satisfy the interests of producers and farmers. Popularization of GAP for TCM herbs depends on the initiative of producers, guidance of the government, and interest of farmers.

\section{Acknowledgement}

$\nabla$

The authors thank Dr. Caixiang Xie for her expert assistance and critical reading in preparation of the manuscript.

\section{References}

1 Ren $D Q$ Zhou RH. Good agricultural practice (GAP) implementation guide for Chinese Crude Drug. Beijing: China Agricultural Press; 2003

2 Qin LP, Huang BK, Zheng HC. The background, present situation, problem and tactics of GAP of Chinese materia medica. J Pharm Pract 2001; 19: 67-70

3 Yao L, Cheng HZ, Yang Z. Guidelines for good agricultural practice of Chinese crude drugs. Beijing: China Agriculture Press; 2006

4 Wei JH, Chen SL, GUO QS. Analysis on GAP implementation of China's current situation and development. Res Inf Tradit Chin Med 2004; 6: 4-8

5 Zheng $Y C$, Bao CY. Industry improved by science and technology-Exhibition of industrial base construction of Jilin traditional Chinese medicine modernization. Beijing: Science and Technology Daily. November 30,2009

6 Chen SL. Sustaining Herbal Supplies: China. Sharing innovative experiences (Vol. 10): examples of the development of pharmaceutical products from medicinal Plants. New York: UNDP; 2005: 157-163

7 Liu S, Wei JH, Chen SL. Study on criteria for GAP certification of wild tending species of Chinese crude drug. Chin Med J Res Pract 2005; 7: 1-4

8 Ran MX. The developmental situation and prospect of planting Chinese material medica in China. Mod Chin Med 2008; 10: 3-6

9 Liu YH. National S \& T development strategy for modernization of traditional Chinese medicine. Mod Tradit Chin Med Mater Med 2005; 7: 1-4

$10 \mathrm{Yu}$ PM, Wang JH, Li H, Zhang N. Probe on the industrialization pattern of Chinese material medica in GAP times. Lishizhen Med Mater Med Res 2007; 17: 665-666

11 Wang JQ Zhang A, Liu YL, Lu DG, Zhang B, Cui JH. Countermeasures and proposals for implement of GAP normalized cultivation of Chinese medicinal materials. Mod Tradit Chin Med Mater Med 2005; 7: 74-77

12 Zhu LX. The relationship research of the Chinese medicine herbs implement GAP cultivation and the superior medicine herbs. Lishizhen Med Mater Med Res 2006; 17: F0003-F0004

13 Liu YM, Feng OJ, Niu X. The characters and problems of Chinese GAP cultivation of traditional medical material. J Shanxi Coll Tradit Chin Med 2002; 3: 46-48

$14 \mathrm{Li} \mathrm{LY}$, Xiao XH, Win SY. Probe on the formation and differentiation of "Dao Di" Chinese crude drug. Chin J Tradit Med Sci Technol 1996; 6: 104-106

15 Huang LQ Zhang RX. Biological investigation on the "Dao Di" Chinese crud drug. Chin Pharm J 1997; 32: 563-566

16 Ding LW. Why did the price of Schisandra chinesis plunge so quickly? The marketing analysis of this species between 2000 and 2010. Mod Chin Med 2009; 11: 48-50

17 Kong L, Qin JX. GAP is a propeller making traditional Chinese medicine into the world. Chin Pharmacol 2003; 12: 7-9 